\title{
AUDIENCIAS CROSSMEDIA: NUEVAS METRICAS Y PERFILES PROFESIONALES EN LOS MEDIOS ESPAÑOLES
}

\section{Crossmedia audiences: New metrics and professional proffles in the Spanish media}

\section{Ana-Isabel Rodríguez-Vázquez, Sabela Direito-Rebollal y Alba Silva- Rodríguez}
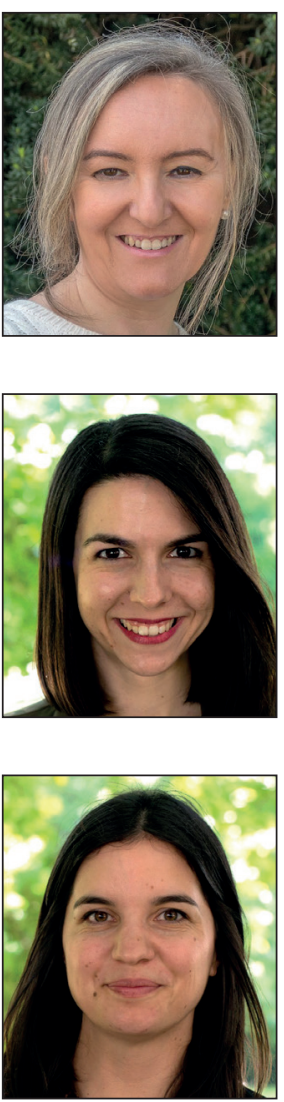

Ana-Isabel Rodríguez-Vázquez es doctora en Comunicación por la Universidade de Santiago de Compostela (USC) y licenciada en Ciencias de la Información por la Universidad Complutense de Madrid (UCM). Imparte materias sobre programación y audiencias e información audiovisual en los grados de Periodismo y Comunicación Audiovisual, y en el master Periodismo y Comunicación: nuevas tendencias. Investigadora del grupo Estudios Audiovisuales (GEA) de la USC, colabora también en proyectos del grupo Novos Medios.

http://orcid.org/0000-0001-7975-1402

anaisabel.rodriguez.vazquez@usc.es

Sabela Direito-Rebollal es graduada en Comunicación Audiovisual por la Universidade de Santiago de Compostela (USC), master en Comunicación e Industrias Creativas (USC) y diplomada en guion de cine y televisión por el Instituto del Cine de Madrid. Es doctoranda en Comunicación e Información Contemporánea en la USC y miembro del grupo de investigación Novos Medios. Su área de estudio se centra en la innovación, programación y audiencias de la televisión pública europea. http://orcid.org/0000-0002-0189-4451

sabela.direito@usc.es

\begin{abstract}
Alba Silva-Rodríguez es doctora en Comunicación por la Universidade de Santiago de Compostela (USC) y licenciada en Periodismo por la misma institución. Imparte materias sobre multimedia y periodismo digital en el grado de Periodismo y en el master Periodismo y Comunicación: nuevas tendencias. Investigadora del grupo Novos Medios de la USC, sus líneas de investigación se centran en el análisis de estrategias, retóricas y formatos tecnológicos para mercados emergentes en la comunicación.
\end{abstract}

http://orcid.org/0000-0002-1221-5178

alba.silva@usc.es

Universidade de Santiago de Compostela Facultade de Ciencias da Comunicación Avenida Castelao, s/n. 15782 Santiago de Compostela (A Coruña), España

\section{Resumen}

La relación del sector infomediario y la medición de audiencias es estrecha: seis de las diez entidades más mencionadas por las empresas españolas como fuentes proveedoras de datos están directamente relacionadas con esta actividad. La convergencia mediática provoca la pérdida de valor de algunos estudios clásicos. Se utilizan fuentes alternativas mientras se perfecciona el consenso en torno a la medición crossmedia. El objetivo de este trabajo consiste en analizar las principales aplicaciones de medición de audiencias que usan los medios españoles con mayor consumo online, así como detectar la existencia de nuevos perfiles profesionales. El estudio se basa en una metodología cuantitativa a partir de encuestas realizadas a once responsables de audiencias y analistas digitales. Los resultados muestran el interés de los medios por monitorizar el comportamiento de sus usuarios, aunque se observan divergencias en las aplicaciones empleadas, en las estructuras organizativas y en las etiquetas con las que designan estos nuevos roles.

\section{Palabras clave}

Audiencias; Métricas; Medios online; Crossmedia; Perfiles profesionales; Participación. 


\begin{abstract}
The relation between infomediary sector and audience measurement is tight: six out of ten entities cited by Spanish companies as data \& information source providers are closely related to this area of audience measurement. The media convergence leads to loss of value of some former studies. Alternative sources are used, whereas the consensus is being improved around the long-awaited measurement model of crossmedia. This article analyses the main audience measurement tools used by those Spanish media with more online readers, as well as checking out the existence of new professional profiles. The research employs a quantitative methodology based on surveys of eleven audience editors and digital analysts. Findings show that media are interested in monitoring the behavior of their users, although divergences are observed in the tools used, in the organizational structures and in the labels with which they name these new roles.
\end{abstract}

\title{
Keywords
}

Audiences; Metrics; Online media; Crossmedia; Professional profiles; Participation.

Rodríguez-Vázquez, Ana-Isabel; Direito-Rebollal, Sabela; Silva-Rodríguez, Alba (2018). “Audiencias crossmedia: nuevas métricas y perfiles profesionales en los medios españoles". El profesional de la información, v. 27, n. 4, pp. 793-800.

https://doi.org/10.3145/epi.2018.jul.08

\section{Introducción y contexto}

Los cambios estructurales -tecnológicos, organizacionales, culturales, políticos, económicos y sociales-a los que se han enfrentado los medios de comunicación en las últimas décadas han influido en el uso que la audiencia hace de ellos (Carpentier; Schrøder; Hallet, 2014). La relación entre los mass media y las audiencias prosumidoras (Toffler, 1981) y comunicativas (Jensen, 2014) crean un nuevo escenario para el intercambio de información (Del-Fresno-García, 2011). Los usuarios se sitúan en el epicentro comunicativo (Nafría, 2017), lo que obliga a los medios a adoptar estrategias para adecuarse a sus gustos, actitudes y motivaciones de consumo, cada vez más orientadas a los dispositivos móviles (comScore, 2018; Deloitte, 2018; Fundación Telefónica, 2018; IAB Spain, 2018; Kemp, 2018; Newman et al., 2017).

Esta etapa de reconversión mediática (Casero-Ripollés, 2012) contempla nuevas rutinas de producción, emisión y consumo (Boczkowski, 2004; Deuze, 2007; MacGregor, 2007; Pavlik, 2010; Rodgers, 2015; Schifferes et al., 2014; Stavelin, 2013). Se demandan profesionales polivalentes que sean capaces de gestionar sistemas de contenido (Rodgers, 2015), algoritmos (Diakopoulos, 2015), audiencias (Tandoc, 2014) o big data (Bruns, 2016). Surgen nuevos nombres para designar estos cometidos: analista y diseñador web, community manager, videoweb editor, editor multimedia y de redes sociales, programmer journalist, experto en usabilidad, engagement editor, editor de audiencias, etc. (Assmann; Diakopoulos, 2017; Flores-Vivar, 2014).

Los nuevos roles exigen paquetes de software analítico (Google Analytics, Adobe Analytics, Chartbeat o Parse.ly) que ofrezcan en tiempo real datos de los visitantes del sitio web, sus preferencias y comportamientos de uso. A estas herramientas se unen las de plataformas de terceros que, como Facebook, son capaces de ofrecer 98 indicadores sobre el perfil de usuario que accede a sus contenidos (Dewey, 2016).

Al poder algorítmico se le atribuye la capacidad de atraer al público mediante listas de recomendaciones (Amazon, YouTube, Netflix, Spotify o Google Play), creadas a partir del rastreo automatizado de los datos de millones de personas
(Manovich, 2018). Las tradicionales métricas de análisis de audiencia deben combinarse con técnicas de big y thick data (Jensen, 2014; Wang, 2013), ya que "confiar únicamente en los big data genera imágenes distorsionadas de los usuarios" y no aporta el valor de contexto necesario (Mondragón-Valero, 2017; Rius, 2017).

\section{Métricas crossmedia: buscando el valor en lo medible}

La distribución online ha permitido que los medios de comunicación midan cómo y qué tipo de contenidos se consumen (Assmann; Diakopoulos, 2017), otorgándoles la posibilidad de complementar los datos ofrecidos por las empresas medidoras con otras fuentes de información (Echegaray; Peñafiel, 2012). Estudios como los de OJD, EGM o Kantar Media conviven ahora con nuevas herramientas de analítica web que rastrean el comportamiento de la audiencia en tiempo real, proporcionando información sobre las páginas vistas o la duración media que los usuarios permanecen en el site (Cherubini; Nielsen, 2016). Estas métricas experimentan su propia revolución en el contexto digital al primar el valor del tiempo sobre el contacto. La automatización y la base algorítmica refuerzan la permanencia frente al tránsito ocasional, de ahí que dos métricas cobren especial relevancia:

- Compromiso: participación de la audiencia

"en función del número de clics (Ci), la duración de la visita (Di), la tasa a la que el visitante regresa al sitio a lo largo del tiempo (Ri), el conocimiento de la marca (Bi), su disposición a contribuir con comentarios ( $\mathrm{Fi}$ ) y la probabilidad de que participen en actividades específicas en el sitio diseñadas para aumentar la conciencia y crear una impresión duradera (Ii)" (Mutter, 2011).

- Recirculación: porcentaje de usuarios que visitan otra web después de consumir un primer contenido. Mide la lealtad de la audiencia y se considera el inverso de la tasa de rebote de Google Analytics,

"el porcentaje de usuarios que abandonaron el sitio web sin cargar una segunda página ni interactuar con nada" (Sweeney, 2018). 
El tiempo de permanencia en el contenido y el número de seguidores del medio se asientan como los pilares de la fidelización online. La nueva ecuación del engagement pasa por la multiplicación del tiempo de lectura, visionado o escucha de un contenido por la frecuencia de retorno y dividido entre todas las plataformas de distribución (móvil, web, etc.).

\section{Medición de audiencias digitales en España}

La digitalización y los nuevos hábitos de los usuarios en distintos dispositivos revolucionan las métricas de audiencias. La distancia actual entre el consumo real de medios y la medición es grande, por lo que es necesario actualizar las herramientas (Sánchez, 2017). La clave de futuro está en obtener un único dato crossmedia que represente adecuadamente el consumo multiplataforma (Santiago, 2017).

En España, la Asociación para la Investigación de los Medios de Comunicación (AIMC), Google, Kantar Media, Facebook, Twitter e IAB Spain figuran entre las diez entidades de referencia de análisis de audiencia, según estudios sobre el sector infomediario (Asedie, 2017; Ontsi, 2017).

Durante 2017, AIMC e IAB Spain convocaron un nuevo concurso para la adjudicación de la medición online. El pliego de condiciones atendía a principios básicos como la población (personas de +4 años, residentes en España y con acceso a internet). En cuanto al tipo de uso se contempla el origen (ordenador de sobremesa o portátil, tableta, smartphone o televisión conectada) y el lugar de consumo (comunidad autónoma, hogar, trabajo o en movilidad) (IAB Spain, 2017). Como aspectos cuantificables se consideran:

- Métricas de audiencia: usuarios únicos, tiempo de consumo, páginas vistas, procedencia del tráfico, tráfico distribuido fuera del sitio principal, visitas y promedio de usuarios únicos diarios.

- Métricas de audio: espectadores únicos, sesiones, reproducciones de audio, tiempo de consumo (emisión en directo o bajo demanda, podcast, etc.), tipo de plataforma (desde el sitio, el player o distribuido en plataformas de terceros como iVoox, iTunes, Tuneln, etc.) y diferenciación entre audio de contenido y publicidad.

- Métricas de vídeo: sesiones, visitas, usuarios únicos, minutos de visionado por visita y usuario, porcentaje medio de visualización y datos de vídeos distribuidos. Por tipo de contenido se medirá la emisión en directo y bajo demanda, el vídeo distribuido en plataformas de terceros (YouTube, Facebook, Dailymotion, etc.) y también se diferenciará entre el vídeo de contenido y el publicitario.

Mientras se resuelve este nuevo concurso ${ }^{1}$ y el sector avanza en el consenso hacia una métrica digital unificada, las empresas emplean herramientas que les permiten aproximarse a sus públicos conectados.

\section{Objetivo y metodología}

El objetivo de este trabajo reside en conocer cuáles son las nuevas herramientas de medición de audiencias en el entorno convergente, así como detectar la existencia de nuevos perfiles profesionales en esta área. Al tratarse de un campo de investigación reciente, se plantea como un estudio exploratorio que busca aproximarse a una realidad aún poco conocida con el fin de detectar prioridades para posteriores investigaciones (Dahnke, 1986).

La metodología empleada se basa en un análisis cuantitativo a partir de un estudio de encuestas. Para delimitar y estandarizar el objeto de estudio, se optó por una muestra de los 20 medios más consumidos por los usuarios, según datos de comscore (noviembre de 2017).

Posteriormente se procedió a la selección de los encuestados en base a su perfil profesional. Se realizó una exploración de los medios escogidos con el fin de localizar, a través de la sección staff, a los encargados del análisis de audiencias. En los casos en los que no fue posible identificarlos se contactó con los responsables de las ediciones digitales para que proporcionaran dicha información. Finalmente, la muestra quedó conformada por los siguientes profesionales:

Tabla 1. Medios más consumidos por los usuarios en internet

\begin{tabular}{|c|c|c|c|c|}
\hline Medios & $\begin{array}{c}\text { Visitantes } \\
\text { únicos (miles) }\end{array}$ & $\%$ reach & $\begin{array}{l}\text { Total de visitas } \\
\text { (millones) }\end{array}$ & $\begin{array}{l}\text { Total de minu- } \\
\text { tos (millones) }\end{array}$ \\
\hline Elpais.com & 19.284 & 60,5 & 391 & 521 \\
\hline Lavanguardia.com & 17.977 & 56,4 & 279 & 515 \\
\hline Elmundo.es & 17.956 & 56,3 & 429 & 638 \\
\hline Abc.es & 16.442 & 51,6 & 210 & 569 \\
\hline Atresmedia & 15.967 & 50,1 & 152 & 658 \\
\hline 20minutos.es & 14.121 & 44,3 & 156 & 192 \\
\hline Marca.com & 13.711 & 43,0 & 401 & 716 \\
\hline Elconfidencial.com & 12.246 & 38,4 & 209 & 409 \\
\hline Mediaset España & 11.742 & 36,8 & 197 & 1.248 \\
\hline As.com & 10.955 & 34,4 & 419 & 426 \\
\hline Hola.com & 10.486 & 32,9 & 107 & 125 \\
\hline Mundodeportivo.com & 9.100 & 28,5 & 118 & 226 \\
\hline Okdiario.com & 8.857 & 27,8 & 95 & 101 \\
\hline Eldiario.es & 8.645 & 27,1 & 73 & 104 \\
\hline Elperiodico.com & 8.611 & 27,0 & 81 & 154 \\
\hline Eleconomista.es & 8.230 & 25,8 & 58 & 109 \\
\hline Rtve & 7.387 & 23,2 & 62 & 422 \\
\hline Huffingtonpost.es & 6.935 & 21,8 & 37 & 46 \\
\hline Expansion.com & 6.846 & 21,5 & 51 & 77 \\
\hline Elespanol.com & 6.830 & 21,4 & 45 & 66 \\
\hline
\end{tabular}

Elaborado con datos de comScore servidos por Barlovento Comunicación 
- Guillermo Castellanos: responsable de audiencias y analítica de El país.

- Francisco Gallego: director del departamento Digital analytics de Unidad Editorial.

- Rubén Vara: director de marketing digital y audiencias multimedia de Atresmedia.

- Alejandro Laso: responsable de innovación y producto en El confidencial.

- Francisco De Campos: responsable de audiencias y analítica digital en As.

- Antonio Rull: encargado de marketing y audiencias de Eldiario.es

- Laura Sanz: directora de producto en El español.

- María Álvarez Carreño: directora de Insights and data marketing de iHola!

- Francisco Morales: analista digital de El periódico.

- Director del departamento de Planificación y Estrategia Comercial Online de El economista.

- Responsable de audiencias de Huffington post.

Se empleó un cuestionario estructurado autoadministrado por email (Bosch; Torrente, 1993), un documento que contemplaba de forma organizada los indicadores de las variables implicadas en el objetivo de la encuesta (Casas-Anguita; Repullo-Labrador; Donado-Campos, 2003). EI cuestionario se envió entre la última semana de enero y la primera de febrero de 2018, y contenía 17 preguntas centradas en tres áreas:

- Organigrama y perfiles profesionales: cuántas personas componían el equipo de analistas de audiencias, qué perfiles profesionales lo integraban y qué etiquetas se empleaban para identificar los nuevos roles (editores de audiencias, analistas de datos, editores de compromiso, etc.).

- Herramientas de trabajo para el análisis de audiencias y conversación social.

- Estrategias de gestión de la participación de la audiencia implantadas por los medios.

\section{Análisis y resultados}

\subsection{Equipos y perfiles profesionales}

La reconfiguración del ecosistema mediático y las actuales dinámicas laborales no sólo han provocado la aparición de renovados perfiles profesionales, sino que también han confirmado una nueva dimensión tecnológica de los mismos. Figuras como analista de audiencias o engagement editor están todavía poco presentes y son también las menos estudiadas desde la academia.

De los medios analizados en este estudio, todos disponen de un equipo de profesionales que trabaja en el área de audiencias. En Eldiario.es, Huffington post y El periódico se ocupa de este cometido una sola persona. Destaca la divergencia a la hora de establecer el nombre de esta nueva figura profesional. En Eldiario.es se le denomina responsable de marketing y audiencias, mientras que en Huffington post es editor de audiencias y en El periódico se conoce como analista digital.
Entre los ocho medios restantes, el 62,5\% dispone de un departamento integrado por tres o cuatro profesionales especializados en periodismo, estadística, SEO, redes sociales y audiencias. El equipo de Unidad Editorial está integrado por 25 personas, mientras que El país cuenta con 20 profesionales en esta área, un tercio de los cuales tiene perfiles técnicos. Todos los medios, excepto El economista -en el que los periodistas trabajan de manera transversal-, contemplan un nombre específico para dicha área. El confidencial, El país y As coinciden en denominarlo Departamento de Desarrollo de Audiencias, mientras que en El español se conoce como Departamento de Audiencia. iHola! y Atresmedia integran a sus analistas de audiencia en el equipo de marketing. En Unidad Editorial existe un área propia que no depende de ningún otro departamento. El resto de medios están subordinados a las siguientes secciones: Dirección General, Estrategia de Producto, Negocio y Desarrollo Digital.

\section{Estudios como los de OJD, EGM o Kantar Media conviven con nuevas herramientas de analítica web que rastrean el compor- tamiento de la audiencia en tiempo real}

\subsection{Herramientas de medición de audiencias}

En un contexto multiplataforma y multipantalla como el actual, es imprescindible disponer de datos objetivos y fiables sobre el comportamiento de los usuarios en el entorno online. Los medios combinan datos proporcionados por los medidores oficiales con el empleo de diferentes softwares que monitorizan la actividad de los usuarios en el site (páginas vistas, tiempo de permanencia, velocidad de lectura, etc.). Para medir el tráfico online, la totalidad de los medios que conforman la muestra emplean los datos de comscore y siete de ellos los de OJDinteractiva. Es el caso de Eldiario.es, El español, El país, Atresmedia y las cabeceras de Unidad Editorial.

Junto a los datos suministrados por estas empresas, todos los medios analizados manejan alguna herramienta complementaria y el 92,3\% -a excepción de Huffington post- utilizan varias. Google Analytics es la que registra un mayor uso (92,3\%), seguida de Adobe Site Catalyst (46,2\%) y Chartbeat $(38,5 \%)$. Parse.ly es la menos popular, ya que sólo Eldiario. es y Huffington post la emplean como mecanismo para determinar el recorrido del usuario a través de los contenidos publicados en sus webs. El $61,5 \%$ de los medios incorporan códigos UTM (Urchin tracking module) en sus urls con el fin de identificar el origen de su tráfico y algunos como El confidencial, El español, Atresmedia o As complementan los datos ofrecidos por este tipo de recursos con la suscripción a otros. Entre los softwares de analítica web, Amplitude es la opción de El confidencial, mientras que As recurre a Similar Web, Welovroi y Tableau. La actividad de los usuarios en el sitio de Atresmedia se monitoriza a través de Crazzy Egg y El español recurre a aplicaciones SEO como Sistrix para optimizar el posicionamiento del diario en los motores de búsqueda.

La utilidad de estos recursos se refleja ya no sólo en el uso que los medios seleccionados hacen de ellos, sino también en la opinión que les merecen a sus responsables de au- 
diencias. El directivo del Departamento de Marketing y Audiencias Digitales de Atresmedia, el de Desarrollo de Audiencias y Analítica de El país y el analista digital de El periódico señalan que todas las herramientas empleadas en sus respectivos medios resultan útiles ya que, como afirma este último, permiten una "medición en tiempo real" y aportan una "medición censal distinta de comscore". Para Guillermo Castellanos (El país),

"forman parte de un ecosistema de medición que sería incompleto si faltase alguna de ellas".

Los medios disponen de un equipo que trabaja en el área de audiencias integrado por profesionales especializados en estadística, SEO y redes sociales

Francisco Gallego (Unidad Editorial) y Francisco De Campos (As) coinciden en que Adobe Site Catalyst es la principal herramienta censal, aunque como afirma el responsable de $A s$,

"para integrar los datos en la redacción Chartbeat ha sido muy útil".

Google Analytics es la preferencia de Eldiario.es, iHola! y El economista,

"por su profundidad" y "porque aporta mucha granularidad", como afirma la responsable del departamento Insights and Data Marketing de iHola! y el directivo del área de Planificación y Estrategia Comercial Online de El economista.

Por su parte, Antonio Rull (Eldiario.es) señala que la principal ventaja de Google Analytics es

"su proyección hacia atrás en el tiempo, facilitando comparativas y mostrando tendencias".

La herramienta más práctica en el trabajo diario de la responsable de audiencias de Huffington post es Parse.ly, aunque en el equipo de desarrollo de audiencias de El confidencial, Amplitude resulta de gran utilidad, ya que como afirma Alejandro Laso,

“permite trabajar por cohortes y así optimizar la retención y recirculación de los usuarios".

Además de medir el tráfico en la web, los medios muestran un creciente interés por analizar la audiencia en los medios socia- les. A excepción de Eldiario.es y El economista, el resto dispone de aplicaciones que permiten valorar el impacto de sus publicaciones en las redes sociales. Entre las más populares destacan Welovroi $(23,1 \%)$, DogTrack $(15,4 \%)$ y Google Analytics (15,4\%). Huffington post, Atresmedia y As optan por el empleo de las utilidades propias de cada plataforma (Twitter Analytics o Facebook Analytics), que combinan con el uso de otras como Social Flow, Ezylnsights o CrowdTangle y Spike para detectar tendencias y monitorizar la actividad de la competencia. Semrush, Buzzsumo y Elephant son las opciones manejadas por El periódico, El confidencial y El español para obtener estadísticas sobre los contenidos (propios o ajenos) que mejor funcionan en las redes sociales y gestionarlas de un modo más efectivo.

El seguimiento de los temas que son tendencia es una prioridad para todos los medios analizados, a excepción de las tres cabeceras de Unidad Editorial. Google Trends (53,8\%) y Trending Topic de Twitter (38,5\%) son las herramientas más empleadas, seguidas de CrowdTangle y Spike (15,4\%). El periódico es el diario que emplea un mayor número de mecanismos para detectar los temas más buscados en las redes sociales, ya que además de Google Trends y Trending Topic de Twitter, utiliza otros como Ezylnsights, Trendsmap o Trendinalia.

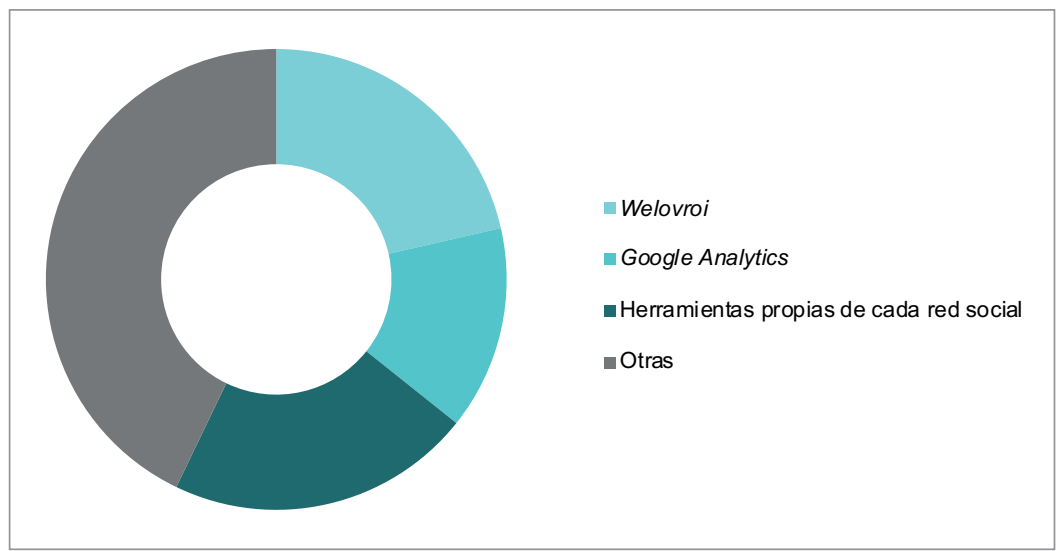

Gráfico 1. Herramientas de medición del impacto de las publicaciones en redes sociales 


\subsection{Gestión de la participación de la audiencia}

La relación de los mass media con su público se ha modificado en un escenario en el que los usuarios disponen de las habilidades e instrumentos necesarios para interactuar directamente con los medios, sus periodistas y con la audiencia digital en general. Gestionar eficientemente esta relación se plantea como uno de los principales retos para que los medios de comunicación enriquezcan la experiencia de sus usuarios, favoreciendo su fidelización y engagement.

El registro a las newsletters, los comentarios y los enlaces a redes sociales son una prioridad en todos los casos analizados, seguido del uso de encuestas y la valoración de contenidos publicados. Las restantes herramientas de interactividad son menos frecuentes, ya que sólo $E I$ confidencial y El país permiten la personalización de interfaces y el seguimiento de temas, mientras que Eldiario.es es el único que tiene foro. El periódico y El confidencial aceptan el envío de contenidos a través de blogs -opción disponible también en El español- y apuestan por el empleo del user generated content -al igual que iHola!-. La creación de una comunidad como vía de conexión y diálogo con el público no es una estrategia para ninguno de los medios estudiados, aunque El confidencial, As o El país ya experimentan con la interacción entre usuarios y bots.

Los medios emplean softwares como Google Analytics, Adobe Site Catalyst y Chartbeat para monitorizar la actividad de los usuarios en el site

Para medir la participación de la audiencia, cinco de los trece medios objeto de estudio disponen de algún software de análisis de los datos, entre los que destacan Google Analytics y Adobe Analytics. La primera es usada por El pe- riódico y la revista iHola!, que complementa la medición con SurveyMonkey para examinar los resultados de las encuestas lanzadas por el medio. El país y As emplean Adobe Analytics, mientras que El confidencial opta por el desarrollo de aplicaciones propias.

\section{Gestionar eficientemente la relación en- tre los medios y la audiencia se plantea como uno de los principales retos para favorecer su fidelización y engagement}

\section{Discusión y conclusiones}

La investigación sobre audiencias necesita reformular sus sistemas de medición para adaptarse al escenario convergente. Este estudio se plantea como una primera aproximación para detectar los nuevos roles profesionales que están incorporando los medios a sus equipos de trabajo y las herramientas que emplean, al margen de las oficiales, para medir la conexión con las audiencias crossmedia. Los datos aportados por los once profesionales encuestados ofrecen una perspectiva cuantitativa sobre cómo los medios de comunicación españoles están afrontando esta reconversión, identificando las coincidencias y divergencias tanto en la organización de sus equipos de trabajo como en el uso de software para rastrear a las audiencias.

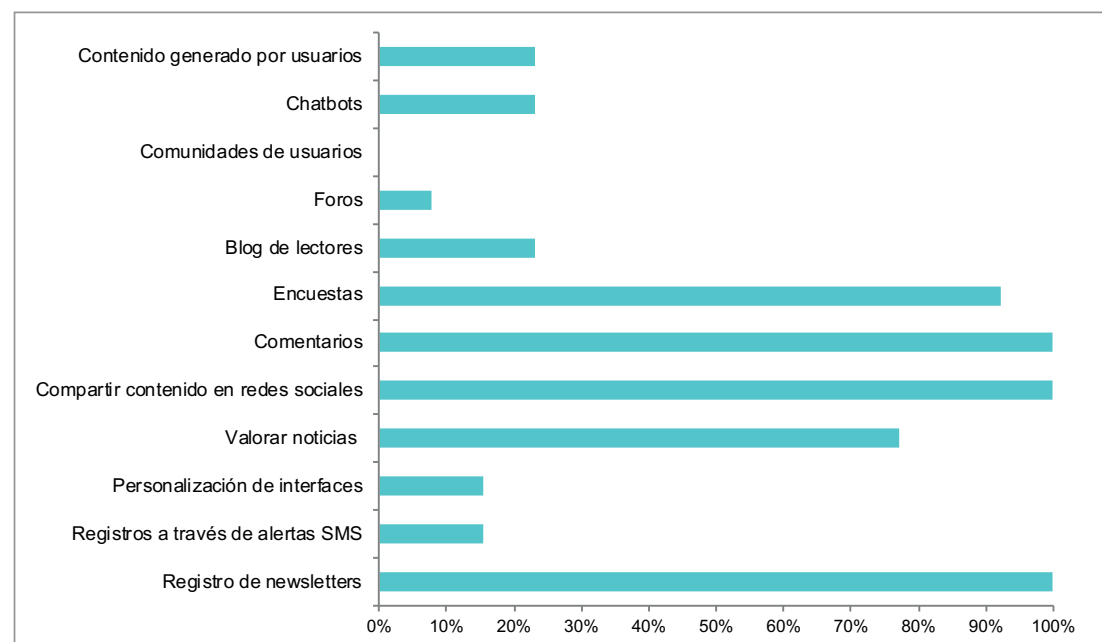

Gráfico 3. Herramientas de interacción con los usuarios
Los resultados muestran que, a nivel operativo, la preocupación por monitorizar el comportamiento de los usuarios es una realidad urgente. De los trece medios estudiados, la mayoría dispone de departamentos de análisis de audiencias integrados por profesionales especializados en estadística, SEO y redes sociales. Unidad Editorial cuenta con un área independiente para este cometido, mientras que en el resto están subordinados a la dirección o a otras secciones. Existe una preocupación por incorporar nuevos perfiles profesionales con competencias tecnológicas y habilidades en el análisis de datos, aunque los medios no coinciden ni en sus estructuras or- 
ganizativas, ni en las etiquetas que usan para designar estos nuevos roles.

El poder del algoritmo queda de manifiesto en el uso que hacen los medios españoles de una amplia gama de herramientas de medición del tráfico digital, de diseño propio o de desarrolladores externos, complementando a la consensuada comScore. El uso de Google Analytics, Adobe Site Catalyst, Chartbeat o de códigos UTM en sus urls concentra las preferencias de los medios estudiados para identificar el origen de su tráfico online y conocer el comportamiento de los usuarios en la web y en las redes sociales.

El tiempo de permanencia en el contenido y el número de seguidores se asientan como los nuevos pilares de la fidelización online. Se pasa del volumen al valor en un ecosistema digital donde cada vez se piden menos clicks y más clocks, donde el coste por mil (CPM) deja paso al coste por horas (CPH) y métricas como el compromiso o la recirculación cobran protagonismo.

A nivel operativo, la preocupación por monitorizar el comportamiento de los usuarios es una realidad urgente

En este contexto, es necesario complementar los datos cuantitativos con otros cualitativos. La tendencia es reforzar los equipos de audiencias de los medios con perfiles de analistas que sepan ir más allá de la cifra. Combinar los big data con los thick data es imprescindible: sin interpretar el contexto y las emociones, el dato pierde valor. El futuro está en la integración de fuentes para alcanzar una métrica digital unificada, avanzar hacia un único dato crossmedia y saber analizarlo desde un enfoque cualitativo para mejorar la conexión de los medios con sus públicos.

\section{Nota}

1. El 11 de abril de 2018, la Mesa de contratación para el concurso de medición de las audiencias digitales resolvió valorar la propuesta de comScore para mantener su contrato con opción de prórroga hasta el año 2023. La resolución se hará firme si el medidor se compromete a asumir las recomendaciones de la Mesa. De momento continúan las negociaciones "de cara a establecer una hoja de ruta que determine el alcance y los plazos de cumplimiento de las exigencias", como señala AIMC (2018).

\section{Agradecimientos}

Este texto está elaborado en el marco del proyecto Usos y preferencias informativas en el nuevo mapa de medios en España: modelos de periodismo para dispositivos móviles (referencia CSO2015-64662-C4-4-R), financiado por el Ministerio de Economía y Competitividad del Gobierno de España y cofinanciado por el fondo estructural Feder. Así mismo, forma parte de las actividades desarrolladas por la Red Internacional de Investigación de Gestión de la Comunicación (Xescom), apoyada por la Consellería de Cultura, Educación e Ordenación Universitaria de la Xunta de Galicia (referencia ED341D R2016/019).
La autora Sabela Direito-Rebollal es beneficiaria del programa para la Formación del Profesorado Universitario (FPU15/02557), financiado por el Ministerio de Educación, Cultura y Deporte del Gobierno de España.

\section{Referencias}

AIMC (2018). comScore, medidor mejor valorado en el concurso para la medición digital en España, 11 de abril. https://goo.gl/fd4pW8

Asedie (2017). Sector infomediario (5a ed.). Asociación multisectorial de la información.

https://goo.gl/wYXcXP

Assmann, Karin; Diakopoulos, Nicholas (2017). “Negotiating change: Audience engagement editors as newsroom intermediaries". In: International symposium on online journalism (ISOJ), pp. 25-44.

https://goo.gl/HeUjha

Boczkowski, Pablo J. (2004). Digitizing the news: innovation in online newspapers. Cambridge: MIT Press. ISBN: 97802 6225461

Bosch, Josep-Lluís; Torrente, Diego (1993). Encuestas telefónicas y por correo. Madrid: CIS. ISBN: 9788474761849

Bruns, Axel (2016). "Big data analysis". En: Witschge, Tamara; Anderson, Christopher; Domingo, David; Hermida, Alfred. The sage handbook of digital journalism. London: Sage, pp. 509-528. ISBN: 9781473906532

Carpentier, Nico; Schrøder, Christian; Hallett, Lawrie (2014). "Audience/society transformations". En: Carpentier, Nico; Schrøder, Christian; Hallett, Lawrie. Audience transformations: Shifting audience positions in late modernity. New York: Routledge, pp. 1-12. ISBN: 9780415827362

Casas-Anguita, Juana; Repullo-Labrador, José-Ramón; Donado-Campos, Juan (2003). "La encuesta como técnica de investigación. Elaboración de cuestionarios y tratamiento estadístico de los datos". Atención primaria, v. 31, n. 8, pp. 527-538. https://doi.org/10.1016/S0212-6567(03)79222-1

Casero-Ripollés, Andreu (2012). "Contenidos periodísticos y nuevos modelos de negocio: evaluación de servicios digitales". El profesional de la información, v. 21, n. 4, pp. 341-346. https://doi.org/10.3145/epi.2012.jul.02

Cherubini, Federica; Nielsen, Rasmus K. (2016). Editorial analytics: How news media are developing and using audience data and metrics. Oxford: Reuters Institute for the Study of Journalism. Digital News Project 2016. https://goo.gl/xERcPP

comScore (2018). Global digital future in focus 2018. https://goo.gl/ajNDqW

Dahnke, Gordon (1986). "Investigación y comunicación". En: Fernández-Collado, Carlos; Dahnke, Gordon. La comunicación humana en ciencia social. México: McGraw-Hill, pp. 385-454. ISBN: 9789684515192

Del-Fresno-García, Miguel (2011). “Infosociabilidad: monitorización e investigación en la web 2.0 para la toma de decisiones". El profesional de la información, v. 20, n. 5, pp. 548-554. https://doi.org/10.3145/epi.2011.sep.09 
Deloitte (2018). Technology, media and telecommunications predictions 2018.

https://goo.gl/P6Vxn2

Deuze, Mark (2007). Media work. London: Polity Press. ISBN: 9780745639246

Dewey, Caitlin (2016). "98 personal data points that Facebook uses to target ads to you". The Washington Post, August $19^{\text {th }}$.

https://goo.gl/2oMvwk

Diakopoulos, Nicholas (2015). “Algorithmic accountability: journalistic investigation of computational power structures". Digital journalism, v. 3, n. 3, pp. 398-415.

https://goo.gl/XoPRh1

https://doi.org/10.1080/21670811.2014.976411

Echegaray, Lázaro; Peñafiel, Carmen (2012). “La utilización de las redes sociales como nuevas herramientas aplicadas al análisis de audiencia". Trípodos, n. 33, pp. 157-172.

https://goo.gl/pCz1Rq

Flores-Vivar, Jesús (2014). Nuevos medios, perfiles y modelos de negocio en la Red. Lima: Universidad San Martín de Porres. ISBN: 9786124221170

Fundación Telefónica (2018). Sociedad digital en España 2017.

https://goo.gl/PBjd2w

IAB Spain (2017). Petición de ofertas de servicios de la medición digital para el mercado español.

https://goo.gl/BgBwGc

IAB Spain (2018). Top tendencias digitales 2018. https://goo.gl/gPkbbV

Jensen, Klaus B. (2014). "Audiences, audiences everywhere - measured, interpreted and imagined". In: Patriarche, Geoffroy; Bilandzic, Helena; Jensen, Jakob L.; Jurišić, Jelena. Audience research methodologies: Between innovation and consolidation. New York: Routledge, pp. 227-239. ISBN: 978 0415827355

Kemp, Simon (2018). “Digital in 2018: World's Internet users pass the 4 billion mark". We are social, Jan. $30^{\text {th }}$. https://goo.gl/q2diFZ

MacGregor, Phil (2007). "Tracking the online audience: Metric data start a subtle revolution". Journalism studies, v. 8, n. 2, pp. 280-298.

https://doi.org/10.1080/14616700601148879

Manovich, Lev (2018). "100 billion data rows per second: Media analytics in the early $21^{\text {st }}$ century". International journal of communication, v. 12, pp. 473-488.

http://ijoc.org/index.php/ijoc/article/viewFile/6160/2249

Mondragón-Valero, Pablo (2017). "Thick data, o por qué Netflix contrata antropólogos". Antropología 2.0, 28 agosto. https://goo.gl/pSmDkK

Rius, Maite (2017). “El poder del thick data en la era del big data". La vanguardia, 10 junio.

https://goo.gl/47w9ud
Mutter, Alan (2011). "Engagement: the new digital metric". Reflections of a newsosaur, Oct. $13^{\text {th }}$. https://goo.gl/k9GGZG

Nafría, Ismael (2017). "Los usuarios, en el centro de la estrategia de los medios". Telos, n. 108, pp. 22-24. https://goo.gl/ErY47m

Newman, Nic; Fletcher, Richard; Kalogeropoulos, Antonis; Levy, David A. L.; Nielsen, Rasmus K. (2017). Digital news report 2017. Oxford: Reuters Institute for the Study of Journalism. https://goo.gl/86hB1o

ONTSI (2017). Estudio de caracterización del sector infomediario en España. Edición 2016. Observatorio Nacional de las Telecomunicaciones y de la Sociedad de la Información. https://goo.gl/3XRCbz

Pavlik, John (2010). "The impact of technology on journalism". Journalism studies, v. 1, n. 2, pp. 229-237.

https://doi.org/10.1080/14616700050028226

Rodgers, Scott (2015). "Foreign objects? Web content management systems, journalistic cultures and the ontology of software". Journalism, v. 16, n. 1, pp. 16-26.

http://eprints.bbk.ac.uk/9506

https://doi.org/10.1177/1464884914545729

Sánchez, Poli (2017). "La medición de audiencias, en la encrucijada". Ipmark: información de publicidad y marketing, 12 abril.

http://ipmark.com/medios-de-comunicacion-medicion-audiencia

Santiago, Fernando (2017). Fragmentación de la audiencia: retos de la medición. Barcelona: Asociación para la Investigación de Medios de Comunicación, AIMC. https://goo.gl/T7Xcgg

Schifferes, Steve; Newman, Nic; Thurman, Neil; Corney, David; Göker, Ayse; Martin, Carlos (2014). "Identifying and verifying news through social media: Developing a user-centred tool for professional journalists". Digital journalism, v. 2, n. 3, pp. 406-418.

https://doi.org/10.1080/21670811.2014.892747

Stavelin, Eirik (2013). Computational journalism: when journalism meets programming. Tesis doctoral. University of Bergen. https://core.ac.uk/download/pdf/30921627.pdf

Sweeney, Andrew (2018). "Why recirculation is a key metric for news publishers in 2018". Mediashift, Dec. $12^{\text {th }}$. https://goo.gl/zRLqZE

Tandoc, Edson C. (2014). "Journalism is twerking? How web analytics is changing the process of gatekeeping". New media \& society, v. 16, n. 4, pp. 559-575. https://doi.org/10.1177/1461444814530541

Toffler, Alvin (1981). La tercera ola. Barcelona: Plaza \& Janés. ISBN: 9788401459306

Wang, Tricia (2013). "Big data needs thick data". Ethnography matters, May 13.

http://ethnographymatters.net/blog/2013/05/13/big-dataneeds-thick-data 